\title{
Commentary: Dazed and malperfused: Predicting outcomes after acute type $A$ aortic dissection repair
}

Leora B. Balsam, MD, ${ }^{\mathrm{a}}$ and Abe DeAnda, Jr, MD ${ }^{\mathrm{b}}$

\author{
From the ${ }^{\mathrm{a} D i v i s i o n}$ of Cardiac Surgery, UMass Memorial Medical Center, Worcester, Mass; and ${ }^{\mathrm{b}}$ Division of Car- \\ diovascular and Thoracic Surgery, University of Texas Medical Branch-Galveston, Galveston, Tex. \\ Disclosures: Authors have nothing to disclose with regard to commercial support. \\ Received for publication Nov 4, 2018; accepted for publication Nov 5, 2018; available ahead of print Jan 7, 2019. \\ Address for reprints: Leora B. Balsam, MD, Division of Cardiac Surgery, UMass Memorial Medical Center, 55 \\ Lake Ave North, Worcester, MA 01655 (E-mail: leora.balsam@umassmemorial.org). \\ J Thorac Cardiovasc Surg 2019;157:1334-5 \\ $0022-5223 / \$ 36.00$ \\ Copyright (C) 2018 by The American Association for Thoracic Surgery \\ https://doi.org/10.1016/j.jtcvs.2018.11.029
}

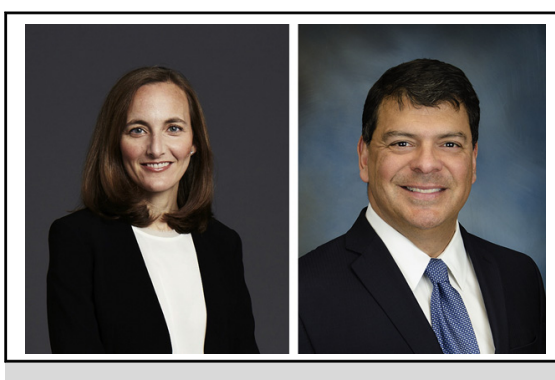

Leora B. Balsam, MD and Abe DeAnda, Jr, MD

Central Message

A new study from the NORCAAD investigators sheds light on malperfusion and its contribution to mortality after surgical repair of acute type A dissection.

See Article page 1324. ischemia: (1) Class Aa, no ischemia, (2) Class Ab, localized ischemia, (3) Class Ac, generalized ischemia, and (4) Class Abc, both localized and generalized ischemia. Olsson and colleagues ${ }^{2}$ reported a mortality of $14 \%$ for Penn Class Aa, 24\% for Penn Class Ab, 24\% for Penn Class Ac, and 44\% for Penn Class Abc in their single-center study of ATAAD repair in Sweden. Others have focused on the relationship between operative mortality and the number of malperfused organs. The Investigators for the German Registry for Acute Aortic Dissection Type A reported a 10\% increase in 30-day mortality for each malperfused organ system. ${ }^{3}$ In this contemporary registry of 2137 patients with ATAAD repair, 30-day mortality was $12.6 \%$ in the absence of malperfusion, $21.3 \%$ for 1 -system malperfusion, $30.9 \%$ for 2-system malperfusion, and $43.4 \%$ for 3 -system malperfusion.

In this issue of the Journal, Zindovic and colleagues ${ }^{4}$ evaluate the impact of malperfusion in the Nordic Consortium for Acute Type A Aortic Dissection (NORCAAD) Registry. The study includes 1159 patients undergoing central aortic repair at 8 Nordic centers between 2005 and 2014. Preoperative malperfusion occurred in $32.9 \%$ of patients and was associated with a higher 30 -day mortality $(28.9 \%$ in patients with malperfusion vs $12.1 \%$ in those without). Early mortality risk segregated as expected when patients were stratified by Penn class. Early mortality was also higher as the number of malperfused organ systems increased $(27.6 \%$ for 1 system, $26.1 \%$ for 2 systems, and $52 \%$ for 3 or more systems). In the long term, patients with malperfusion had significantly worse survival, largely because of their increased early

hazard of death. The NORCAAD experience is similar to that of other large registries, including the German Registry for Acute Aortic Dissection Type A and the International Registry of Acute Aortic Dissection. The overall message continues to be that malperfusion increases the risk of mortality with ATAAD.

Measures of malperfusion are often included in risk models for mortality with ATAAD. For example, Rampoldi and colleagues ${ }^{5}$ and the International Registry of Acute Aortic Dissection investigators developed a risk model that incorporates clinical measures of malperfusion, including pulse deficits and electrocardiographic findings of myocardial ischemia or infarction. Leontyev and colleagues $^{6}$ also developed a "score card" to predict in-hospital death after ATAAD repair, assigning increasing weight for coronary, extremity, and visceral malperfusion. ${ }^{6}$ Because of its large size, the NORCAAD dataset would be useful for further validation of these models.

The preferred treatment for ATAAD with malperfusion is a matter of debate. Conventional treatment is emergency central aortic repair, which reestablishes antegrade true lumen flow and prevents proximal aortic rupture. The Stanford group has advocated for initial aortic repair and reported similar in-hospital and midterm survival in patients with and without malperfusion treated in this manner. Zindovic and colleagues ${ }^{4}$ do not clarify whether adjunctive procedures were performed for malperfusion in their series (before, during, or after central aortic repair), and this is an 
important missing piece of information. It highlights a general weakness of registry data, a lack of case-by-case granularity.

Time and again, the cannulation technique has come up when considering the approach to ATAAD repair in the setting of malperfusion. NORCAAD investigators ${ }^{4}$ used a myriad of techniques, with no subgroup analysis reported to help understand what works best. In general, it is expected that expert aortic surgeons will modify their technique in the presence of malperfusion; however, this NORCAAD report does not clarify how and when this was done. Whether this represents a real-world experience remains unanswered.

When considering malperfusion in ATAAD, a differentiation must be made between dynamic and static malperfusion. In dynamic malperfusion, the motion of the intimal flap causes obstruction of a branch vessel and end-organ ischemia. In static malperfusion, branch vessel obstruction occurs, often caused by true lumen compression by a thrombosed false lumen. The treatment for dynamic and static malperfusion is different; dynamic malperfusion often resolves with central aortic repair, whereas static malperfusion typically does not. In those situations, intravascular fenestration, true lumen stenting, or extraanatomic bypass may be required. Again, the NORCAAD registry report does not add to our understanding of how to best handle these cases. Some groups have advocated for revascularization of malperfused beds first and delayed central aortic repair. The Michigan group ${ }^{8}$ has used this approach consistently since 1997, with 70 of 173 patients $(40 \%)$ with ATAAD undergoing percutaneous (endovascular-first) treatment for suspected malperfusion. In their series, $33 \%$ of the endovascular-first patients died while awaiting central aortic repair, half as the result of aortic rupture and the other half as the result of ongoing complications of malperfusion. Critics of the Michigan approach focus on the significant early mortality from aortic rupture, which may have been prevented with earlier central aortic repair. Other groups have described strategies for rapid restoration of perfusion to malperfused organs, followed by immediate central aortic repair. Uchida and colleagues $^{9}$ from Japan used percutaneous coronary intervention for coronary malperfusion, surgical fenestration for carotid artery occlusion, active perfusion of the superior mesenteric artery via laparotomy for visceral malperfusion, and brachial artery to femoral artery temporary bypass for lower-extremity malperfusion in their series of 438 patients with ATAAD; these reperfusion strategies were followed by immediate central aortic repair. Malperfused patients treated with this strategy had a remarkable early mortality of only $3.6 \%$, compared with
$18 \%$ for patients with malperfusion who received central aortic repair only.

Not all malperfusion is created equal, or is it? The group at Washington University ${ }^{10}$ reported that a preoperative base deficit less than or equal to -10 was associated with a $92 \%$ operative mortality. Malperfusion of certain organs seems worse than others, with visceral malperfusion having the highest mortality in most studies. Time to resolution of malperfusion is clearly linked to outcomes as well. The NORCAAD registry data shed some light on this topic, even though information regarding the specific treatment for malperfusion is not given. In their multivariable analysis, Zindovic and colleagues ${ }^{4}$ found that any malperfusion, cardiac malperfusion, renal malperfusion, and peripheral malperfusion were associated with 30-day mortality to varying degrees. The authors also found that any malperfusion, cardiac malperfusion, and visceral malperfusion predicted late mortality.

No two dissections are the same, so repairing them forces the surgeon to make patient-specific adjustments. Although surgical evidence suggests that most patients do well with emergency central aortic repair, the challenge is deciding how to best manage the others. This report from the NORCAAD investigators corroborates the first point but leaves the second point unanswered.

\section{References}

1. Augoustides JG, Geirsson A, Szeto WY, Walsh EK, Cornelius B, Pochettino A, et al. Observational study of mortality risk stratification by ischemic presentation in patients with acute type A aortic dissection: the Penn classification. Nat Clin Pract Cardiovasc Med. 2009;6:140-6.

2. Olsson C, Hillebrant CG, Liska J, Lockowandt U, Eiksson P, Franco-Cereda A. Mortality in acute type A aortic dissection: validation of the Penn classification. Ann Thorac Surg. 2011;92:1376-83.

3. Czerny M, Schoenhoff F, Etz C, Englberger L, Khaladj N, Zierer A, et al. The impact of pre-operative malperfusion on outcome in acute type A aortic dissection: results from the GERAADA registry. J Am Coll Cardiol. 2015;65:2628-35.

4. Zindovic I, Gudbjartsson T, Ahlsson A, Fuglsang S, Gunn J, Hansson EC, et al. Malperfusion in acute type A aortic dissection: an update from the Nordic Consortium for Acute Type A Aortic Dissection. J Thorac Cardiovasc Surg. 2019;157:1324-33.e6.

5. Rampoldi V, Trimarchi S, Eagle KA, Nienaber CA, Oh JK, Bossone E, et al. Simple risk models to predict surgical mortality in acute type A aortic dissection: the international registry of acute aortic dissection score. Ann Thorac Surg. 2007;83:55-61.

6. Leontyev S, Legare JF, Borger MA, Buth KJ, Funkat AK, Gerhard J, et al. Creation of a scorecard to predict in-hospital death in patients undergoing operations for acute type A aortic dissection. Ann Thorac Surg. 2016;101:1700-6.

7. Chiu P, Tsou S, Goldstone AB, Louie M, Woo YJ, Fischbein MP. Immediate operation for acute type A aortic dissection complicated by visceral or peripheral malperfusion. J Thorac Cardiovasc Surg. 2018;156:18-24.

8. Patel HJ, Williams DM, Dasika NL, Suzuki Y, Deeb GM. Operative delay for peripheral malperfusion syndrome in acute type A aortic dissection: a long-term analysis. J Thorac Cardiovasc Surg. 2008;135:1288-96.

9. Uchida K, Karube N, Kasama K, Minami T, Yasuda S, Goda M, et al. Early reperfusion strategy improves the outcomes of surgery for type A acute aortic dissection with malperfusion. J Thorac Cardiovasc Surg. 2018;156:483-9.

10. Lawton JS, Moon MR, Liu J, Koerner DJ, Kulshrestha K, Damiano RJ Jr, et al The profound impact of combined severe acidosis and malperfusion on operative mortality in the surgical treatment of type A aortic dissection. J Thorac Cardiovasc Surg. 2018;155:897-904. 